\title{
Influence of deformed potentials on elastic scattering reactions involving deformed projectile-spherical target
}

\author{
Murat AYGÜN a,* \\ a Bitlis Eren University, Department of Physics, TR-13000, Bitlis Turkey
}

\author{
ART IC LE INFO \\ Article history: \\ Received 17 December 2020 \\ Received in revised form 26 January 2021 \\ Accepted 25 January 2021 \\ Keywords: \\ Deformation and orientation \\ Elastic scattering \\ Optical model
}

\begin{abstract}
A B S TRACT
We investigate the effects of potentials with quadrupole and hexadecapole deformations on the elastic scattering angular distributions of deformed projectiles. We calculate the elastic scattering cross-sections of ${ }^{20} \mathrm{Ne}+{ }^{12} \mathrm{C},{ }^{22} \mathrm{Ne}+{ }^{12} \mathrm{C},{ }^{24} \mathrm{Mg}+{ }^{24} \mathrm{Mg}$ and ${ }^{28} \mathrm{Si}+{ }^{27} \mathrm{Al}$ reactions by using Deformed Broglia-Winther (1991) (DBW91), Deformed Aage Winther (DAW95) and Deformed Woods-Saxon (DWS) potentials. Then, we perform the calculations for different orientation angles such as $\theta=0$ and $\theta=\pi / 2$ of deformed projectiles. Finally, we compare the theoretical results together with the experimental data.
\end{abstract}

(C) 2021. Turkish Journal Park Academic. All rights reserved.

\section{Introduction}

Elastic scattering cross-sections were analyzed extensively for spherical states of nuclei. Different methods including various density distributions [1-3] and nuclear potentials [4-6] were used in order to explain experimental data. However, deformation effect was generally neglected in the calculations. If one or both of nuclei are deformed, the effect of deformation and orientation of nuclei should be included in the analysis of nuclear interactions. In this context, the deformation effect on nuclear reactions was investigated with different studies [7-9]. It is known that the theoretical calculations with deformed case are more difficult. Therefore, different approaches can be found from the literature. One of these is to use a deformed potential. For this purpose, there are different deformed potentials in the literature. As a result, the question of which potential is more valid arises. Thus, we believe that it will be important and useful to investigate the suitability of different deformed potentials for deformed projectiles.

We investigate the effect of deformed potentials on the elastic scattering cross-sections of deformed projectiles. We calculate the elastic scattering cross-sections of $20 \mathrm{Ne}+{ }^{12} \mathrm{C},{ }^{22} \mathrm{Ne}+{ }^{12} \mathrm{C}$, ${ }^{24} \mathrm{Mg}+{ }^{24} \mathrm{Mg}$ and ${ }^{28} \mathrm{Si}+{ }^{27} \mathrm{Al}$ reactions for three various deformed potentials such as Deformed Broglia-Winther (1991) (DBW91), Deformed Aage Winther (DAW95) and Deformed Woods-Saxon (DWS). Then, we chose two important orientation angles such as $\theta=0$ and $\theta=\pi / 2$ where the nucleus can be found. We repeat the theoretical calculations for $\theta=0$ and $\theta=\pi / 2$ angles of deformed projectiles. We compare our results with the data.

Section 2 gives calculation process. Section 3 provides information about deformed potentials. Section 4 shows the results and discussion. Finally, Section 5 is assigned to the summary and conclusion.

\section{Calculation procedure}

The total potential for deformed projectile and spherical target can be expressed as

$U(r)=V_{c}(r)+V(r)+i W(r)$

where $V_{c}(r), V(r)$ and $W(r)$ are the Coulomb, real and imaginary potentials, respectively. $V c(\mathrm{r})$ potential is [10]

\footnotetext{
* Corresponding author. Tel.: +90 (434) 222 0020; fax: +90 (434) 2229143

E-mail address: maygun@beu.edu.tr

ORCID : 0000-0002-4276-3511 (M. Aygün)
} 


$$
\begin{aligned}
V_{C}(r) & =\frac{1}{4 \pi \varepsilon_{0}} \frac{Z_{P} Z_{T} e^{2}}{r}, \quad r \geq R_{C} \\
& =\frac{1}{4 \pi \varepsilon_{0}} \frac{Z_{P} Z_{T} e^{2}}{2 R_{C}}\left(3-\frac{r^{2}}{R_{C}^{2}}\right), \quad r<R_{C} \\
R_{c} & =1.25\left(A_{P}^{\frac{1}{3}}+A_{T}^{\frac{1}{3}}\right) .
\end{aligned}
$$

$V(r)$ is calculated using DBW91, DAW95 and DWS potentials which are described below. $W(r)$ is assumed as

$$
W(r)=-\frac{W_{0}}{1+\exp \left(\frac{r-r_{W}\left(A_{P}^{\frac{1}{3}}+A_{T}^{\frac{1}{3}}\right)}{a_{W}}\right)}
$$

where $W_{0}, r_{w}$ and $a_{w}$ are the depth, radius and diffuseness parameters, respectively. The elastic scattering cross-sections are acquire via the code FRESCO [11].

\section{Deformed potentials}

\subsection{Deformed Broglia-Winther 1991 (DBW91) potential}

The DBW91 potential can be written in the following form

$$
V_{N}^{D B W 91}(r, \theta)=-\frac{V_{0}(\theta)}{\left[1+\exp \left(\frac{r-R_{0}(\theta)}{a}\right)\right]} \mathrm{MeV},
$$

Where

$$
\begin{aligned}
& V_{0}(\theta)=16 \pi \frac{R_{P}(\theta) R_{T}}{R_{P}(\theta)+R_{T}} \gamma a, \\
& R_{0}(\theta)=R_{P}(\theta)+R_{T}+0.29 \\
& R_{i}(\theta)=\left(1.233 A_{i}^{1 / 3}-0.98 A_{i}^{-1 / 3}\right)\left[1+\beta_{20} Y_{20}(\theta)+\beta_{40} Y_{40}(\theta)\right](i=P, T)
\end{aligned}
$$

$P$ and $T$ subscripts indicate the projectile and target, respectively. The $\beta_{2}$ and $\beta_{4}$ values which are traditionally used to symbolize nuclear deformation show quadrupole and hexadecapole deformation parameters of deformed projectiles respectively, and are given in Table 1 [12].

Table 1. The $\beta_{2}$ and $\beta_{4}$ deformation values of deformed projectiles.

\begin{tabular}{lcc}
\hline Projectile & $\beta_{2}$ & $\beta_{4}$ \\
\hline${ }^{20} \mathrm{Ne}$ & 0.364 & 0.207 \\
${ }^{22} \mathrm{Ne}$ & 0.384 & 0.096 \\
${ }^{24} \mathrm{Mg}$ & 0.393 & -0.012 \\
${ }^{28} \mathrm{Si}$ & -0.363 & 0.187 \\
\hline
\end{tabular}

\subsection{Deformed Aage Winther (DAW95) potential}

The DAW95 potential is similar to the DBW91 potential except for [13]

$a=\left[\frac{1}{1.17\left(1+0.53\left(A_{P}^{-1 / 3}+A_{T}^{-1 / 3}\right)\right)}\right] \mathrm{fm}$,

and
$R_{0}(\theta)=R_{P}(\theta)+R_{T}$

With

$R_{P}(\theta)=\left(1.2 A_{P}^{1 / 3}-0.09\right)\left[1+\beta_{20} Y_{20}(\theta)+\beta_{40} Y_{40}(\theta)\right], R_{T}=1.2 A_{T}^{1 / 3}-0.09$

\subsection{Deformed Woods-Saxon (DWS) potential}

The DWS potential is assumed as [14]

$V_{N}^{D W S}(r, \theta)=-\frac{V_{0}}{\left[1+\exp \left(\frac{r-R(\theta)}{a}\right)\right]} \mathrm{MeV}$,

Where

$R(\theta)=1.17+R_{P}\left[1+\beta_{20} Y_{20}(\theta)+\beta_{40} Y_{40}(\theta)\right]+R_{T} \quad(i=P, T)$

(12)

$$
R_{P(T)}=\left(1+0.39 I_{P(T)}\right) A_{P(T)}^{1 / 3}, I_{P(T)}=\frac{N_{P(T)}-Z_{P(T)}}{A_{P(T)}}, a=\left[0.5+0.33\left(I_{P}+I_{T}\right)\right] \mathrm{fm}
$$

$I_{P(T),} Z_{P(T)}, N_{P(T)}$ and $A_{P(T)}$ are isospin asymmetry, atomic number, neutron number and mass number of projectile(target), respectively. The potential depth $V_{0}$ is evaluated as [15]

$$
V_{0}=-44.16\left[1-0.40\left(I_{P}+I_{T}\right)\right] \frac{A_{P}^{\frac{1}{3}} A_{T}^{\frac{1}{3}}}{A_{P}^{\frac{1}{3}}+A_{T}^{\frac{1}{3}}}
$$

\section{Results and Discussion}

A comparative analysis of deformed potentials that can be used to explain the elastic scattering cross-sections between deformed projectile and spherical target nuclei was performed. However, it is also possible to consider target nuclei and Coulomb potential as deformed. Thus, they may give also contributions to the cross-sections. Here we investigated the effect of deformation on the projectile without considering other deformed situations. While studying the deformation effect of the projectiles, we assumed their ground states. With this goal, each potential was achieved by using FORTRAN code which was written by us. Then, the FRESCO cards over the acquiring results were formed. As a result of this, the real part of the optical model potential was obtained. In Fig. 1, we showed the distance-dependent changes of the real potentials of deformed different projectile reactions at $\theta=0$ and $\theta=\pi / 2$ orientations. We observed that the real potentials of DBW91 and DAW95 potentials behaved similarly for ${ }^{20} \mathrm{Ne},{ }^{22} \mathrm{Ne}$ and ${ }^{24} \mathrm{Mg}$ nuclei except for ${ }^{28} \mathrm{Si}$. The real potential depths of ${ }^{20 \mathrm{Ne}}$, ${ }^{22} \mathrm{Ne}$ and ${ }^{24} \mathrm{Mg}$ nuclei decrease with increasing orientation angle while the depth of ${ }^{28} \mathrm{Si}$ nucleus increases. It was found that the DWS potential was the deepest for all nuclei and all deformed potentials. The effect of the orientation angle for the DWS potential was observed in the tail part of the potential compared to other potentials. In addition, we noticed that this tailing showed a different situation for the ${ }^{28}$ Si nucleus. 

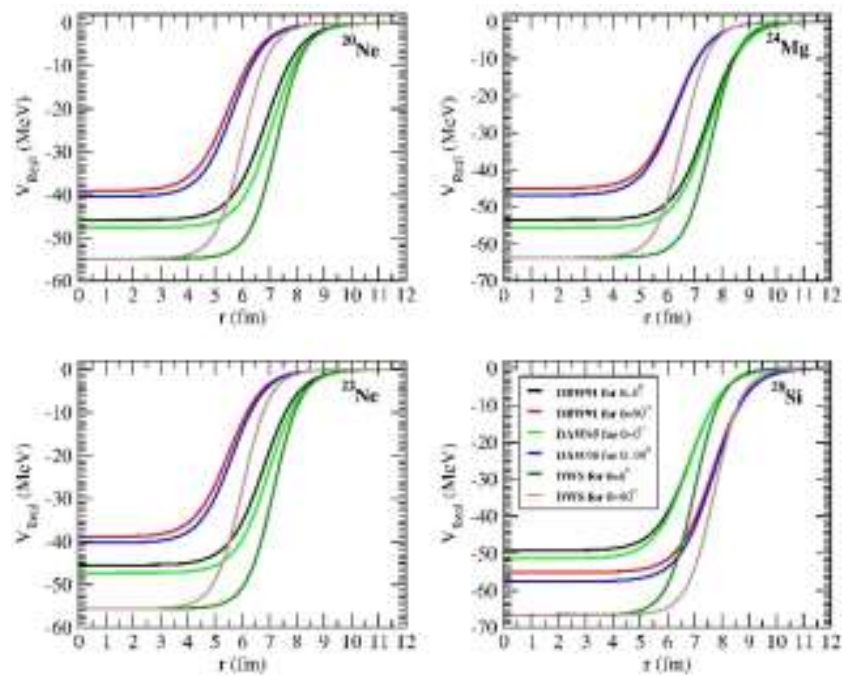

Figure 1. Distance-dependent changes of the real potentials of deformed projectile and spherical target nucleus reactions at different orientations.

The Woods-Saxon potential was applied for the imaginary part of the optical model. The $r_{w}$ and $a_{w}$ parameters were investigated in steps of $0.1 \mathrm{fm}$ and $0.01 \mathrm{fm}$, and the $W_{0}$ value was determined for convenient results with the data. Thus, the potential parameters of the reactions were given in Table 2.

Table 2. The $w_{0}$ (in $\mathrm{MeV}$ ), $r_{w}$ (in $\mathrm{fm}$ ), $a_{w}$ (in $\mathrm{fm}$ ) values used in the calculations of the elastic scattering cross-sections of ${ }^{20} \mathrm{Ne}+{ }^{12} \mathrm{C},{ }^{22} \mathrm{Ne}+$ ${ }^{12} \mathrm{C},{ }^{24} \mathrm{Mg}+{ }^{24} \mathrm{Mg}$ and ${ }^{28} \mathrm{Si}+{ }^{27} \mathrm{Al}$ reactions with DBW91, DAW95 and DWS potentials and at $\Theta=0$ and $\Theta=\frac{\pi}{2}$ orientations.

\begin{tabular}{|c|c|c|c|c|c|c|c|}
\hline \multirow[t]{2}{*}{ Reaction } & \multirow[t]{2}{*}{ Parameter } & \multicolumn{2}{|c|}{ DBW91 } & \multicolumn{2}{|c|}{ DAW95 } & \multicolumn{2}{|l|}{ DWS } \\
\hline & & $\Theta=0$ & $\Theta=\frac{\pi}{2}$ & $\Theta=0$ & $\Theta=\frac{\pi}{2}$ & $\Theta=0$ & $\Theta=\frac{\pi}{2}$ \\
\hline \multirow{3}{*}{${ }^{20} \mathrm{Ne}+{ }^{12} \mathrm{C}$} & $W_{0}$ & 84.0 & 39.5 & 95.0 & 43.0 & 105.0 & 48.0 \\
\hline & $r_{w}$ & 1.38 & 1.38 & 1.38 & 1.38 & 1.38 & 1.38 \\
\hline & $a_{w}$ & 0.88 & 0.88 & 0.88 & 0.88 & 0.88 & 0.88 \\
\hline \multirow{3}{*}{${ }^{22} \mathrm{Ne}+{ }^{12} \mathrm{C}$} & $W_{0}$ & 80.0 & 39.0 & 89.0 & 38.0 & 93.0 & 44.0 \\
\hline & $r_{w}$ & 1.38 & 1.38 & 1.38 & 1.38 & 1.38 & 1.38 \\
\hline & $a_{w}$ & 0.88 & 0.88 & 0.88 & 0.88 & 0.88 & 0.88 \\
\hline \multirow{3}{*}{${ }^{24} \mathrm{Mg}+{ }^{24} \mathrm{Mg}$} & $W_{0}$ & 1.00 & 1.00 & 1.00 & 1.00 & 1.00 & 1.00 \\
\hline & $r_{w}$ & 1.38 & 1.38 & 1.38 & 1.38 & 1.38 & 1.38 \\
\hline & $a_{w}$ & 0.88 & 0.88 & 0.88 & 0.88 & 0.88 & 0.88 \\
\hline \multirow{3}{*}{${ }^{28} \mathrm{Si}+{ }^{27} \mathrm{Al}$} & $W_{0}$ & 1.00 & 1.10 & 1.00 & 1.00 & 1.00 & 1.00 \\
\hline & $r_{w}$ & 1.38 & 1.38 & 1.38 & 1.38 & 1.38 & 1.38 \\
\hline & $a_{w}$ & 0.88 & 0.88 & 0.88 & 0.88 & 0.88 & 0.88 \\
\hline
\end{tabular}

We calculated the elastic scattering cross-sections of $20 \mathrm{Ne}+{ }^{12} \mathrm{C}$ reaction at $390 \mathrm{MeV}$ and ${ }^{22} \mathrm{Ne}+{ }^{12} \mathrm{C}$ reaction at $264 \mathrm{MeV}$ by using DBW91, DAW95 and DWS potentials for $\theta=0$ and $\theta=$ $\pi / 2$. We compared the theoretical results together with the experimental data for ${ }^{20} \mathrm{Ne}+{ }^{12} \mathrm{C}$ in Fig. 2 and for ${ }^{22} \mathrm{Ne}+{ }^{12} \mathrm{C}$ in Fig. 3. We observed that deformed potentials gave an average behavior in explaining the experimental data that exhibit an oscillating structure. In addition, all the potentials for these reactions were seen to produce a marked phase shift at different orientation angles.

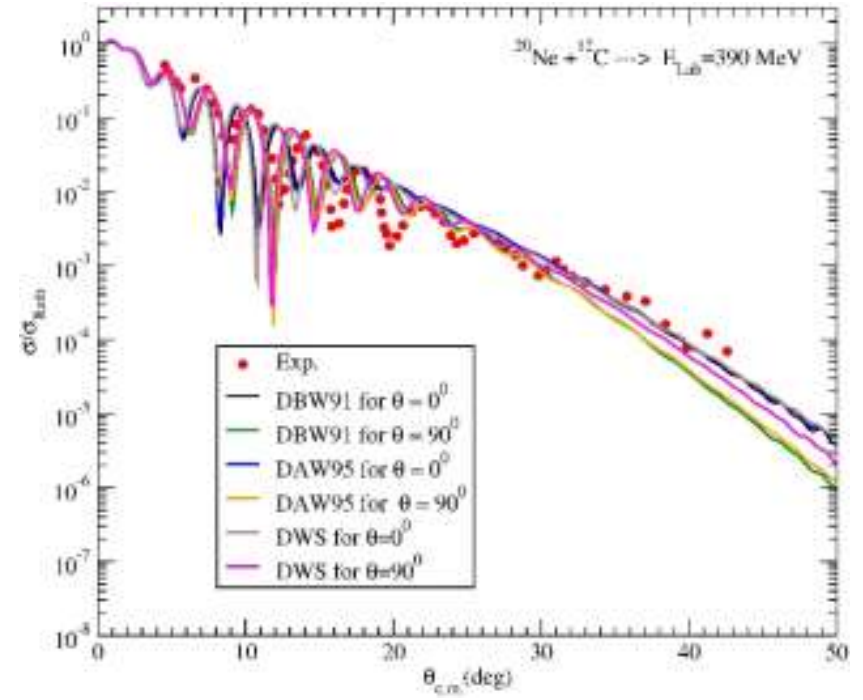

Figure 2. The elastic scattering cross sections of ${ }^{20} \mathrm{Ne}+{ }^{12} \mathrm{C}$ reaction at $390 \mathrm{MeV}$ by using DBW91, DAW95 and DWS potentials for $\theta=0$ and $\theta$ $=\pi / 2$. The data are from Ref. [16].

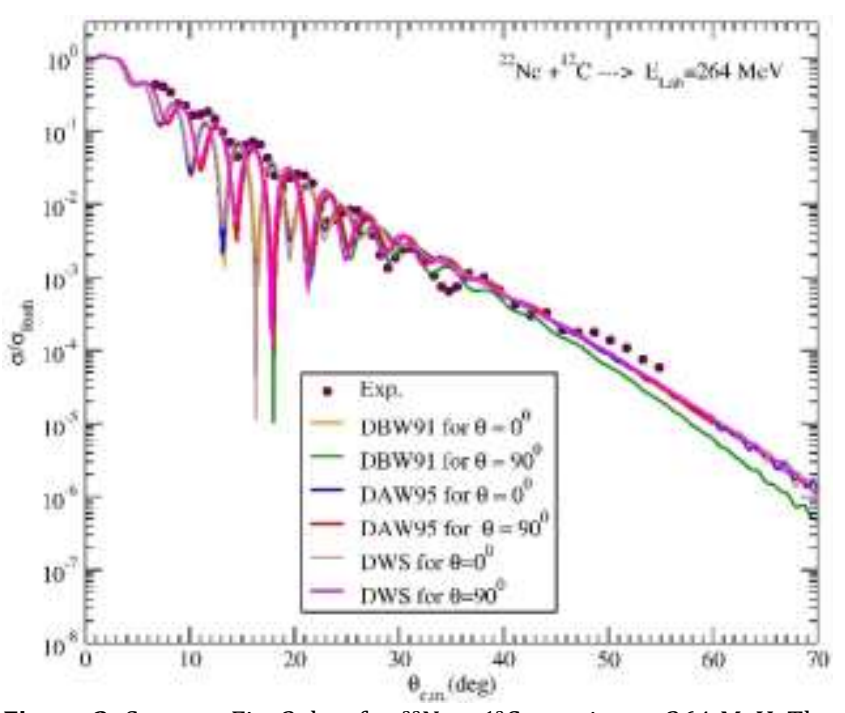

Figure 3. Same as Fig. 2, but for ${ }^{22} \mathrm{Ne}+{ }^{12} \mathrm{C}$ reaction at $264 \mathrm{MeV}$. The data are from Ref. [17].

Then, we obtained the elastic cross-sections of ${ }^{24} \mathrm{Mg}+{ }^{24} \mathrm{Mg}$ reaction at $60 \mathrm{MeV}$ and ${ }^{28} \mathrm{Si}+{ }^{27} \mathrm{Al}$ reaction at $70 \mathrm{MeV}$. The calculations were based on DBW91, DAW95 and DWS potentials for $\theta=0$ and $\theta=\pi / 2$. We compared the theoretical results with the data for ${ }^{24} \mathrm{Mg}+{ }^{24} \mathrm{Mg}$ in Fig. 4 and for ${ }^{28} \mathrm{Si}+{ }^{27} \mathrm{Al}$ in Fig. 5. The potential results for ${ }^{24} \mathrm{Mg}+{ }^{24} \mathrm{Mg}$ reaction cannot explain the initial values of the experimental data. Although they explain some of the later angles, the results are not in very good agreement with the data in general sense. Additionally, the potentials display a phase shift at different orientation angles. For ${ }^{28} \mathrm{Si}+{ }^{27} \mathrm{Al}$ reaction, it is seen that the results of the deformed potentials are not in good agreement with the experimental data. Especially, for $\theta \geq 470$, the potentials are quite inadequate in explaining the experimental data. Also, phase shifts were observed for different orientation angles of the deformed potentials. 


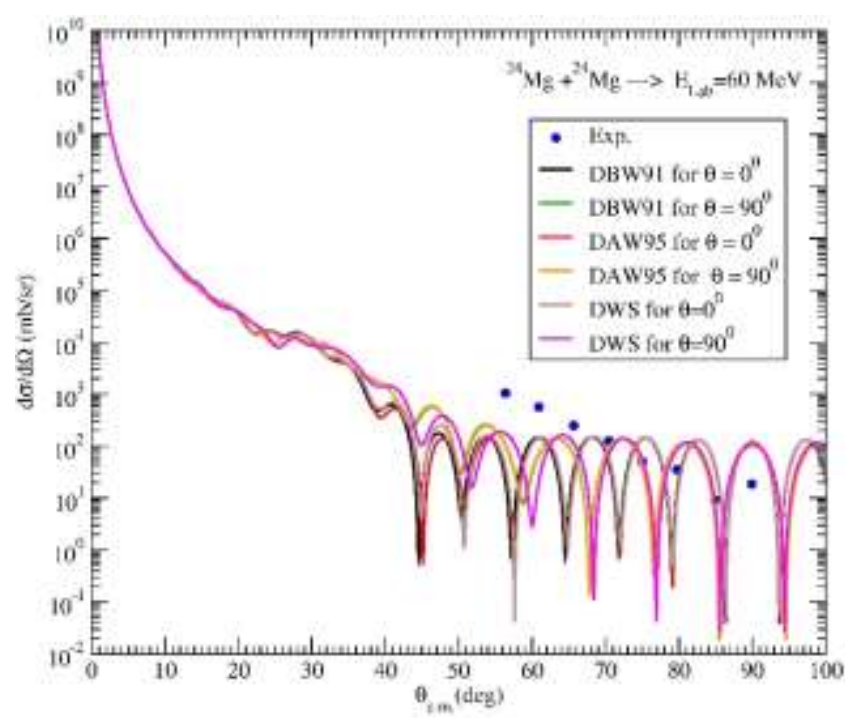

Figure 4. Same as Fig. 2, but for ${ }^{24} \mathrm{Mg}+{ }^{24} \mathrm{Mg}$ reaction at $60 \mathrm{MeV}$. The data are from Ref. [18].

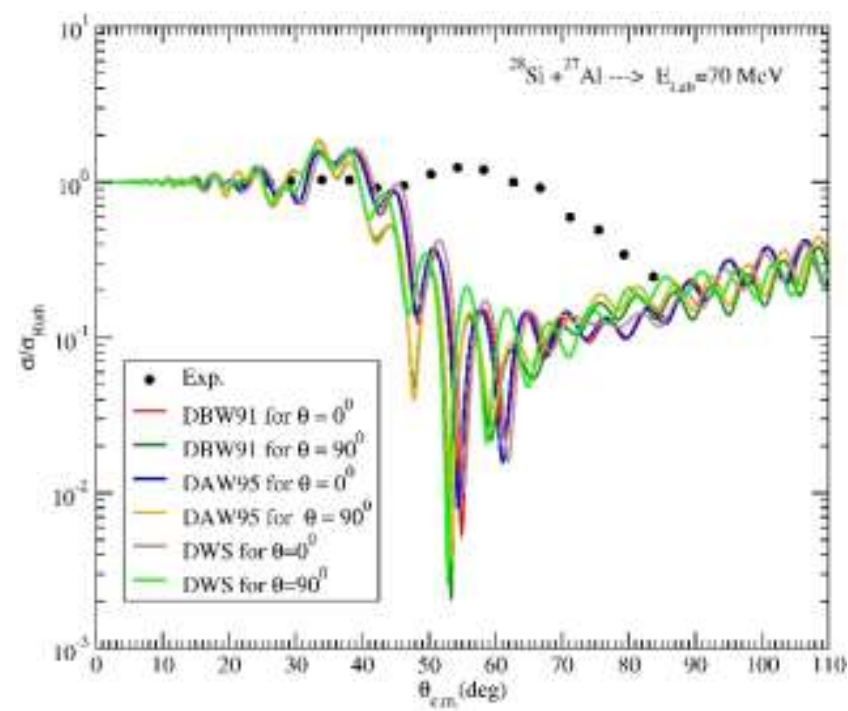

Figure 5. Same as Fig. 2, but for ${ }^{28} \mathrm{Si}+{ }^{27} \mathrm{Al}$ reaction at $70 \mathrm{MeV}$. The data are from Ref. [18].

One of the remarkable results of our study, all the deformed potentials could not provide good agreement results with the elastic scattering experimental data of ${ }^{24} \mathrm{Mg}+{ }^{24} \mathrm{Mg}$ and ${ }^{28} \mathrm{Si}+$ ${ }^{27} \mathrm{Al}$ reactions although they gave consistent results in defining the elastic scattering experimental data of ${ }^{20} \mathrm{Ne}+{ }^{12} \mathrm{C}$ and ${ }^{22} \mathrm{Ne}$ $+{ }^{12} \mathrm{C}$ reactions. In other words, although the deformed potentials are different from each other, the results were seen to be similar. We think that the structural conditions of the projectiles are more prominent. In this context, we investigated the deformation parameters of all the deformed projectiles. We noticed that the $\beta_{2}$ and $\beta_{4}$ values of ${ }^{20 \mathrm{Ne}}$ and ${ }^{22} \mathrm{Ne}$ nuclei are positive, whereas the $\beta_{2}$ and $\beta_{4}$ values of ${ }^{24} \mathrm{Mg}$ and ${ }^{28} \mathrm{Si}$ nuclei are negative. That is, we consider that quadrupole and hexadecapole deformations play a key role in the theoretical calculations.

\section{Summary and Conclusions}

In our study, the effect of three different deformed potentials such as DBW91, DAW95 and DWS potentials on the analysis of elastic scattering angular distributions of deformed projectile and spherical target was investigated. In this context, although the results of potentials is compatible with the experimental data for ${ }^{20} \mathrm{Ne}$ and ${ }^{22} \mathrm{Ne}$ nuclei, the same results could not be obtained for ${ }^{24} \mathrm{Mg}$ and ${ }^{28} \mathrm{Si}$ nuclei. Especially, the results of $28 \mathrm{Si}$ nucleus were seem to be incompatible with data. We concluded that the structural properties of the deformed projectiles were found to be more dominant than the shapes of potentials. Finally, we noticed that the orientation of deformed projectiles affected the shape of the nucleus-nucleus potential well. Therefore, we believe that the deformed potentials used to explain the scattering cross sections of the deformed nuclei should be investigated for further nuclear interactions.

\section{References}

[1] Aygun, M., 2018. Analysis with SDHO and RMF density distributions of elastic scattering cross-sections of oxygen isotopes $\left({ }^{16-18} 0\right)$ by various target nuclei. International Journal of Modern Physics E, 27, 1850055.

[2] Aygun, M., 2019. Analysis with relativistic mean-field density distribution of elastic scattering cross-sections of carbon isotopes $\left({ }^{10-14,16} \mathrm{C}\right)$ by various target nuclei. Pramana - Journal of Physics, 93, 72.

[3] Aygun, M., Aygun, Z., 2019. Microscopic analysis of elastic scattering cross sections for different densities of ${ }^{8} \mathrm{Li}$ nucleus on light, medium and heavy mass targets. Revista Mexicana de F'isica, 65, 404-411.

[4] Aygun, M., 2018. Alternative Potentials Analyzing the Scattering Cross Sections of $7,9,10,11,12,14 \mathrm{Be}$ Isotopes from a ${ }^{12} \mathrm{C}$ target: Proximity Potentials. Journal of the Korean Physical Society, 73, 1255-1262.

[5] Aygun, M., 2018. The application of some nuclear potentials for quasielastic scattering data of the ${ }^{11} \mathrm{Li}+{ }^{28} \mathrm{Si}$ reaction and its consequences. Turkish Journal of Physics, 42, 302-311.

[6] Aygun, M., 2018. A Comparison of Proximity Potentials in the Analysis of Heavy-Ion Elastic Cross Sections. Ukrainian Journal of Physics, 63, 881-887.

[7] Rashdan, M., Sewailem, Sh. M., 2019. Deformation and orientation effects on reaction cross-sections at intermediate and high energies. International Journal of Modern Physics E, 28, 1950014.

[8] Rashdan, M., 2012. Deformation, orientation, and medium effects in ${ }^{16,19} \mathrm{C}+\mathrm{C}$ reactions. Physical Review C 86, 044610.

[9] Hassan, M.Y.M., Farag, M.Y.H., Abul-Magd, A.Y., Nassar, T.E.I., 2008. Nucleus-nucleus reaction cross sections for deformed nuclei. Physica Scripta,78, 045202.

[10] Satchler, G.R., 1983. Direct Nuclear Reactions. Oxford University Press.

[11] Thompson, I. J., 1988. Coupled reaction channels calculations in nuclear physics. Computer Physics Reports, 7, 167.

[12] Möller, P., Sierk, A.J., Ichikawa, T., Sagawac, H., 2016. Nuclear ground-state masses and deformations: FRDM(2012). Atomic Data and Nuclear Data Tables, 109-110, 1-204.

[13] Winther, A., 1995. Dissipation, polarization and fluctuation in grazing heavy-ion collisions and the boundary to the chaotic regime. Nuclear Physics A, 594, 203-245.

[14] Pahlavani, M.R., Alavi, S.A., Tahanipour, N., 2013. Effect of nuclear deformation on the potential barrier and alpha-decay half-lives of superheavy nuclei. Modern Physics Letters A, 28, 16. 
[15] Wang, N., Scheid, W., 2008. Quasi-elastic scattering and fusion with a modified Woods-Saxon potential. Physical Review C, 78, 014607.

[16] Bohlen, H.G., Stiliaris, E., Gebauer, B., von Oertzen, W., Wilpert, M., Wilpert, Th., Ostrowski, A., Khoa, D. T., Demyanova, A.S., Ogloblin, A.A., 1993. Refractive scattering and reactions, comparison of two systems: ${ }^{16} \mathrm{O}+{ }^{16} \mathrm{O}$ and ${ }^{20} \mathrm{Ne}+{ }^{12} \mathrm{C}$. Zeitschrift fur Physik A, 346, 189-200.

[17] Al-Abdullah, T., Carstoiu, F., Chen, X., Clark, H. L., Fu, C., Gagliardi, C. A., Lui, Y.-W., Mukhamedzhanov, A., Tabacaru, G., Tokimoto, Y., Trache, L., Tribble, R. E., 2010. Physical Review C, 81, 035802.

[18] https://www-nds.iaea.org/exfor/ 